\title{
Multiple views of magnetism in cool stars
}

\author{
J. Morin ${ }^{1, \star}$, M. Jardine ${ }^{2}$, A. Reiners ${ }^{1}$, D. Shulyak ${ }^{1}$, B. Beeck ${ }^{1}$, G. Hallinan ${ }^{3}$, L. Hebb ${ }^{4}$, G. Hussain ${ }^{5}$, \\ S. V. Jeffers ${ }^{1}$, O. Kochukhov ${ }^{6}$, A. Vidotto $^{2}$, and L. Walkowicz ${ }^{7}$ \\ 1 Institut für Astrophysik, Georg-August-Universität Göttingen, Friedrich-Hund Platz, 37077 Göttingen, Germany \\ 2 School of Physics and Astronomy, Univ. of St Andrews, St Andrews, Scotland KY16 9SS, UK \\ 3 California Institute of Technology, 1200 E. California Blvd., Pasadena, CA 91125, USA \\ 4 Department of Physics and Astronomy, Vanderbilt University, Nashville, TN 37235, USA \\ 5 ESO, Karl-Schwarzschild-Str. 2, D-85748 Garching, Germany \\ 6 Department of Physics and Astronomy, Uppsala University Box 516, 75120 Uppsala, Sweden \\ 7 Department of Astrophysical Sciences, Princeton University, Princeton, NJ 08544, USA
}

Received XXXX, accepted XXXX

Published online XXXX

Key words stars: low-mass, brown dwarfs, stars: magnetic fields, stars: activity, stars: coronae, stars: starspots

\begin{abstract}
Magnetic fields are regarded as a crucial element for our understanding of stellar physics. They can be studied with a variety of methods which provide complementary - and sometimes contradictory - information about the structure, strength and dynamics of the magnetic field and its role in the evolution of stars. Stellar magnetic fields can be investigated either with direct methods based on the Zeeman effect or through the observation of activity phenomena resulting from the interaction of the field with the stellar atmosphere. In this Cool Stars XVII Splinter Session we discussed the results obtained by the many ongoing studies of stellar activity and direct studies of surface magnetic fields, as well as the stateof-the-art techniques on which they are based. We show the strengths and limitations of the various approaches currently used and to point out their evolution as well as the interest of coupling various magnetism and activity proxies.
\end{abstract}

\section{Introduction}

Since the first detection on the Sun through the Zeeman effect in 1908 by G. E. Hale, magnetic fields have become a key element of stellar physics. They are now thought to play an important role throughout the formation and evolution of stars and their planetary systems. In addition, they are now detected across a large part of the H-R diagram (e.g. Donati \& Landstreet 2009; Reiners 2012), either directly or through the activity phenomena they power which can be observed across a large part of the electromagnetic spectrum. The characterization of these magnetic fields has revealed that stars show a very wide variety of magnetic properties in terms of strength, geometry or variability. Drawing a clear picture of stellar magnetism and dynamo processes is however not easy since different types of measurements do not always agree - at least apparently - and have led to various interpretations (e.g. Reiners et al. 2009; Morin et al. 2011 in the case of M dwarfs).

In this session, we discussed a number of approaches used to detect, measure and model stellar magnetic fields as well as the related activity phenomena. A particular emphasis was put on the comparison of different approaches or proxies, the way they can be combined, and on the ongoing developments in instrumentation, analysis and modelling.

* Corresponding author:e-mail: jmorin@gwdg.de

\section{Surface magnetic field and starspots}

\subsection{Starspots from spectroscopy and spectropolarimetry}

Direct measurements of stellar magnetic fields at the photospheric level rely on the properties of the Zeeman effect. Two complementary techniques are successfully employed. On the one hand, Zeeman-induced line broadening measured in unpolarized light directly provides a measurement of the average surface field modulus - weighted e.g. by limb-darkening and brightness inhomogeneities. This approach can be applied regardless of the field complexity, but it only provides very little information about the field geometry. Such measurements are limited to low $v \sin i$ values (up to $\sim 20 \mathrm{~km} / \mathrm{s}$ ). On the other hand, the measurement of polarisation in spectral lines is sensitive to the vector properties of the surface magnetic field. However this technique can only probe the large-scale structure of stellar magnetic fields - due to the cancellation of polarised signal corresponding to neighbouring regions of opposite polarities and is blind to bipolar groups for instance.

Doppler imaging (DI) is a powerful tool for investigating long-lived stellar surface features on rapidly-rotating stars (Vogt \& Penrod 1983). It relies on the correspondence between the spatial position of a surface inhomogeneity on a rotating star and the wavelength of the corresponding distortion in the Doppler-broadened line profile. It allows to re- 
trieve the locations, shapes and sizes of starspots. M. Semel (1989) first proposed to extend DI to polarised spectra in order to investigate magnetic fields and termed this new technique "Zeeman-Doppler Imaging" (ZDI). In particular, ZDI can disentangle between field orientations, and allowed to demonstrate the existence of magnetic fields quite different from the Sun's, for instance in young solar-type stars with shallow convective envelopes (Jeffers \& Donati 2008).

\subsection{Seeing Spots: Observations of Stellar Magnetic Activity with Kepler}

Although Kepler's primary mission is the detection of extrasolar planets, its incredible precision and high cadence provide an exquisite opportunity to study the interplay between stellar rotation - both global and differential - and magnetic activity. These observations allow us to place our Sun in the context of other stars, and improve not only our understanding of the stars themselves, but the range of environments in which planets exist, a crucial element to model their surfaces and atmospheres.

The Kepler mission has now obtained over three years of nearly contiguous observations for Sun-like stars. These lightcurves allow to determine rotation periods, measure differential rotation and detect flares on a wide variety of stars, both similar to and much cooler than the Sun. With such a wealth of new information it is now possible to make new advances on the rotation-activityrelation across the H-R diagram (e.g. Basri et al. 2011); or to derive stellar rotation axis inclinations (when combined with $v \sin i$ measurements, Buchave et al. in prep.). The combination of rotation period measurements with asteroseismic diagnostics derived from Kepler ligh-curves also bears the potential of extending gyrochronology calibrations to slowly rotating stars. Ongoing efforts to characterize stellar variability and activity phenomena in the Kepler target stars include modelling of spot distributions, detections of differential rotation and flare statistics (e.g., Walkowicz et al. 2011; Walkowicz, Basri \& Valenti, submitted).

\subsection{Understanding the effect of magnetic fields on the fundamental properties of low mass stars through ZDI and eclipsing binary modelling of YY Gem}

A multi-faceted analysis of the magnetic field properties and global fundamental parameters of the well known $\mathrm{M}$ dwarf eclipsing binary YY Gem (e.g. Torres \& Ribas 2002) is ongoing. Understanding the magnetic field properties of YY Gem is important because it is one of the few systems that provide a benchmark for stellar evolution models of early $\mathrm{M}$ dwarfs, and may allow us to understand how activity affects stellar structure (see e.g. Hebb et al. 2012).

Through a coordinated observing campaign, coincident high precision multi-band light curves and time series spectropolarimetry of the system were obtained over 10 consecutive nights in January 2012. Preliminary magnetic field maps and surface brightness maps derived from the ZDI analysis of both components of the system were derived. The resulting surface brightness maps are incorporated as constraints on the starspot parameters when completing a new eclipsing binary modelling. In this way, precise and accurate masses and radii could be derived for both stars. The updated value of the discrepancy between observed and theoretical mass-radius relation is about $5 \%$, lower than previous estimates. Yet more eclipsing binary systems must be analysed to to solve this long-standing problem of massradius relation in active low-mass stars.

Finally, a preliminary analysis of the frequency and flux of the optical flares that were observed during the photometric campaign was performed. By combining these multiple analyses, a first global picture of the large-scale magnetic field properties of two early M dwarf stars can be drawn. This study ultimately aims to assess the effect of the magnetic field on the fundamental parameters of cool stars.

\subsection{Can we map starspots and their magnetic fields using infra-red lines and molecular bands?}

Currently employed methods of spectropolarimetric analysis of cool active stars - such as Least-Squares Deconvolution combined with ZDI (cf. Sec. 2.1) - are systematically missing strong magnetic fields inside cool starspots since they do not take into account temperature inhomogeneities. It is widely believed that an extension of polarimetric studies to molecular bands and magnetically sensitive infra-red lines may allow detection and mapping of starspot magnetic fields. Magnetometry in the near infra-red is indeed attractive due to the wavelength dependence of the Zeeman effect and the reduced spot-to-photosphere intensity contrast. Atomic lines in the near infra-red however tend to be shallower than in the visible. Zeeman-sensitive molecular lines - mainly TiO (7055 $\AA$ ) and FeH (9900 $)$ - on their side can prove valuable to probe magnetic fields inside cool spots, although the amplitude polarization signal in these lines is lower than in Fe I lines (see e.g. Shulyak et al. 2010).

These possibilities are tested with comprehensive ZDI simulations based on the methodology presented by Kochukhov et al. (2009). The calculations demonstrate that neither molecular features nor infra-red atomic lines taken separately provide sufficient information to simultaneously recover the contrast and geometry of starspots. Both diagnostics must be combined with optical lines in order to achieve substantial improvement of the magnetic and temperature reconstruction.

\subsection{MHD simulations of surface convection in cool main-sequence stars}

Cool main-sequence stars have thick convective envelopes or are fully convective. Magnetic fields have been detected in many of such stars. In the Sun, the observed surface magnetic field is highly structured owing to its interaction with the convective flows. The local structure of the magnetic 
field in other cool stars is, however, unknown. In the absence of spatially resolved observations, the effect of the local structure of the magnetic field on detectable signals like magnetically sensitive spectral lines can be evaluated by numerical simulations of the magneto-convective processes.

Comprehensive 3D radiative magnetohydrodynamic simulations of the surface layers of main-sequence stars of spectral types F3 to M2 were carried out (see e.g. Vögler et al. 2012). The simulations were analysed in terms of sizes and properties of the convection cells (granules) and magnetic flux concentrations. Synthetic spectral line profiles were generated and the impact of the different magnetic structures of these stars on their spectra was investigated.

These simulations show a qualitative difference in magneto-convection between solar-like stars and $\mathrm{M}$ dwarfs (Beeck et al. 2011). The synthetic spectra yield rough lower limits for the detectability of magnetic fields in cool stars depending on rotation velocity and global field geometry. It is shown that the net effect of magnetic field - broadening, distortion and apparent radial velocity shift - on a Zeemansensitive spectral line is largely degenerate with the star's $v \sin i$ and radial velocity for average field moduli up to $500 \mathrm{G}(50 \mathrm{mT})$. Fitting several spectral lines simultaneously will likely help overcoming this issue. Finally, these results can be used to test magnetic field measurement methods like ZDI, because they provide known field configurations and their time-dependent line spectra.

\section{Coronae and magnetospheres}

\subsection{Understanding stellar coronae: Insights and challenges}

Surface magnetic field maps of cool stars have been used to produce detailed models of the coronae and extended environments of cool stars that can successfully reproduce a range of observational characteristics. These coronal field models are powerful tools that have the potential to explain how a cool star system evolves from the pre-main sequence - when the star is actively accreting from its primordial disk - to the main sequence - when magnetically driven stellar winds control the angular momentum evolution of stars.

Stellar coronae can be studied through their X-ray emission both in quiescent and flaring states. Rotational modulation of X-ray emission can be detected in active binaries, and the X-ray-emitting coronae of active $\mathrm{G}$ and $\mathrm{K}$ dwarfs are shown to be very compact. Current stellar coronal models are generally based on extrapolations of the surface magnetic maps reconstructed with ZDI - thus defining the footpoints of X-ray emitting coronal loops - and assume an isothermal corona in hydrostatic equilibrium. $\mathrm{X}$ ray emission can then be computed assuming an optically thin corona. Such models have been successful at reproducing X-ray rotational modulation (e.g. Hussain et al. 2007) as well as constraining the location of accretion hotspots in T Tauri stars (e.g. Gregory et al. 2010).
One of the challenges faced by such models is the limited spatial resolution of ZDI surface magnetic maps, and their impact on the derived quantities (e.g. Arzoumanian et al. 2011; Jardine et al. in prep.). New methods based on MHD dynamical modelling allow to take into consideration self-consistently the interaction of the outflowing wind with the magnetic field and vice versa (Vidotto et al. 2011). Upcoming observational capabilities such as ALMA will also prove useful in probing circumstellar environments and enabling future advances.

\subsection{Radio Signatures of Magnetospheric Phenomena on Active Stars, Brown Dwarfs, and Extrasolar Planets}

The radio wavelength domain offers powerful tools to characterize stellar and planetary magnetic fields. The actual derivation of magnetic field strength depends on the emission process involved. For the Sun, quiescent emission is dominated by thermal emission from the chromosphere and the corona, whereas non-thermal emission is powered by particle acceleration during impulsive events like flares. Planets on their side produce a very bright low-frequency $(\mathrm{kHz}-\mathrm{MHz})$ emission powered by quasi-stable particle acceleration. VLA observations revealed that many active stars also possess a nonthermal corona where large populations of electrons are continuously accelerated to high energies, a situation with no solar counterpart.

The advent of a new generation of radio observatories, together with the upgrades to existing facilities, offers the potential for significant breakthroughs in the study of magnetospheric phenomena on active stars, brown dwarfs and exoplanets as well as new avenues for synergies with observations in other wavelength regimes. Interferometers, such as the Jansky VLA, now offer the capability of timeresolved wideband spectroscopy enabling the detection and frequency characterization of sweeping radio bursts, a capability previously only available in observations of the Sun.

Future multi-wavelength campaigns will allow the correlation of such bursts with the higher energy counterparts from optical to X-rays. Very Long Baseline interferometry (VLBI) enables the resolved imaging of coronal structures on nearby stars during both quiescence and flare events (e.g. Peterson et al. 2010), which in turn can be directly mapped to the large scale magnetic fields revealed by tomographic techniques such as ZDI. Beyond the main sequence, the detection of auroral, pulsed radio emission provides the sole means for direct determination of magnetic field strengths for cool brown dwarfs (e.g. Hallinan et al. 2007), exemplified by the recent radio detection of a T6.5 dwarf (Route \& Wolszczan 2012). Similar emissions may soon be detectable from extrasolar planets with the advent of the new generation of low frequency arrays, such as the LWA and LOFAR. 


\subsection{Investigating the effects of magnetic cycles on the Tau Boo system}

To quantify the effect stellar cycles have on orbiting planets, one has to understand the properties of the stellar wind, which depend on the particular geometry of the coronal magnetic field at each epoch during the stellar cycle. $\tau$ Boo is an intriguing planet-host star that is believed to undergo magnetic cycles similar to the Sun, but with a duration that is about one order of magnitude smaller than that of the solar cycle. With the use of observationally derived surface magnetic field maps, the magnetic stellar wind of $\tau$ Boo can be studied by means of three-dimensional MHD numerical simulations (Vidotto et al. 2012).

In total, four surface magnetic maps are used, they encompass one full magnetic cycle i.e. two polarity reversals (e.g. Fares et al. 2009). Wind mass loss-rate is found to vary little during the observed epochs of the cycle (less than 3 per cent), with a relatively more important variation in angular momentum loss-rates (a factor of 2). The models show that the emission measure from the quiescent closed-field line corona remains approximately constant through the cycle, in agreement with recent X-ray observations of $\tau$ Boo (Poppenhaeger et al. 2012). This rather cycle-independent X-ray emission from $\tau$ Boo could point to the presence of overlapping activity cycles (e.g., overlap in the butterfly diagram, McIvor et al. 2006).

The rather rapid changes in the magnetic properties of the stellar corona and the relatively high mass-loss rates of $\tau$ Boo $\left(\sim 2.7 \times 10^{-12} \mathrm{M}_{\odot} \mathrm{yr}^{-1}\right.$, i.e., two orders of magnitude larger than that of the solar wind) imply that the environment that surrounds $\tau$ Boo b (and other putative planets in this system) is significantly different from the environment surrounding the planets in the solar system. The radio emission from the hot Jupiter that orbits at 0.0462 au from $\tau$ Boo is estimated. It is shown that, for a planet with a magnetic field similar to Jupiter ( $\sim 14 \mathrm{G}$ at the pole), the radio flux is estimated to be about $1.4 \mathrm{mJy}$, occurring at a frequency of $34 \mathrm{MHz}$, potentially detectable from the ground.

\section{Summary and discussion}

The interpretation and comparison of magnetic field measurements and modelling stemming from different approaches is a debated topic. There is, however, a general agreement that a better knowledge of the spatial structure of stellar magnetic fields is essential in order to reconcile these different views, and that what we know about the Sun likely cannot be safely extrapolated to stars with very different properties (in particular very active stars). For instance, the predominance of bright (plage-like) $v s$ dark (spot-like) regions among magnetic surface features, the very existence of regions corresponding to "quiet" photosphere on very active stars, are of great relevance to magnetic field measurements and start to be addressed through numerical simulations (sec. 2.5). This importance of the spatial structure of the field is even relevant to the vocabulary used. It can be argued that "magnetic field" should be reserved for an actual magnetic field vector at some point of the stellar surface, whereas observationally-derived quantities should be termed "magnetic flux". Both quantities have the same unit of Gauss (CGS) or Tesla (SI), although some confusion can arise with a quantity also called "magnetic flux" and measured in Maxwell (i.e. G.cm², CGS) or Weber (i.e. T.m², SI), see Reiners (2011) and Reiners \& Mohanty (2012).

The talks given in the session suggest that in the next few years progress will arise from the use of new or upgraded observational facilities, coupled with improved modelling methods and from multi-wavelength or multitechnique studies. Ultimately, such advances will aim at a better characterization of stellar magnetic fields and planetary environments, as well as a better understanding of magnetic field generation in cool stars.

Acknowledgements. We thank the organizers of the 17th Cool Stars conference for giving us the opportunity to hold this session. JM acknowledges the support of the Alexander von Humboldt foundation.

\section{References}

Arzoumanian, D., et al.: 2011, MNRAS 410, 2472-2480

Basri, G., et al.: 2011, AJ 141, 20

Beeck, B., Schüssler, M., Reiners, A.: 2011. In Johns-Krull, C., Browning, M. K., West, A. A. (Eds.), AIPCS Vol. 448 (p. 1071)

Donati, J. F., Landstreet, J. D.: 2009, ARAA 47, 333-370

Fares, R., et al.: 2009, MNRAS 398, 1383-1391

Gregory, S. G., Jardine, M., Gray, C. G., Donati, J. F.: 2010, Reports on Progress in Physics 73(12), 126901

Hale, G. E.: 1908, ApJ 28, 315

Hallinan, G., et al.: 2007, ApJL 663, L25-L28

Hebb, L., et al.: 2012. AAS Abstracts, Vol. 219 (p. 345.15)

Hussain, G. A. J., et al.: 2007, MNRAS 377, 1488-1502

Jeffers S. V., Donati J.-F., 2008, MNRAS, 390, 635

Kochukhov, O., et al.: 2009. In Stempels, E. (Ed.), AIPCS Vol. 1094 (pp. 720-723)

McIvor, T. et al.: 2006, MNRAS 367, 592

Morin, J., et al.: 2011, MNRAS 418, L133-L137

Peterson, W. M., et al.: 2010, Nature 463, 207-209

Poppenhaeger, K., et al.: 2012, AN 333, 26

Reiners, A.: 2012, Living Reviews in Solar Physics 9, 1

Reiners, A., Basri, G.: 2009, A\&A 496, 787-790

Reiners, A. and Mohanty, S.: 2012, ApJ 746, 43

Reiners, A.: 2011, In Johns-Krull, C., Browning, M. K., West, A. A. (Eds.), AIPCS Vol. 448 (p. 255)

Route, M., Wolszczan, A.: 2012, ApJL 747, L22

Semel, M.: 1989, A\&A 225, 456-466

Shulyak, D., et al.: 2010, A\&A 523, A37

Torres, G., Ribas, I.: 2002, ApJ 567, 1140-1165

Vidotto, A. A., et al.: 2012, MNRAS 423, 3285-3298

Vidotto, A. A., et al.: 2011, MNRAS 412, 351-362

Vögler A., et al.: 2003, IAUS, 210, 157

Vogt, S. S., Penrod, G. D.: 1983, PASP 95, 565-576

Walkowicz, L. M., et al.: 2011, AJ 141, 50 